\title{
Verbascoside-A Review of Its Antitumor Activities
}

\author{
Hasan Alaa Aldeen Khalaf', Ruaa Azziz Jasim¹, Ismail Taha Ibrahim ${ }^{2,3}$ \\ ${ }^{1}$ Pharmacognosy Department, College of Pharmacy, Al-Bayan University, Baghdad, Iraq \\ ${ }^{2}$ Pharmacology Department, College of Pharmacy, Al-Bayan University, Baghdad, Iraq \\ ${ }^{3}$ Hot Laboratory, Egyptian Atomic Energy, Cairo, Egypt \\ Email: ismailtaha_73@yahoo.com
}

How to cite this paper: Khalaf, H.A.A., Jasim, R.A. and Ibrahim, I.T. (2021) Verbascoside-A Review of Its Antitumor Activities. Pharmacology \& Pharmacy, 12, 109126.

https://doi.org/10.4236/pp.2021.126011

Received: May 9, 2021

Accepted: June 27, 2021

Published: June 30, 2021

Copyright (c) 2021 by author(s) and Scientific Research Publishing Inc. This work is licensed under the Creative Commons Attribution International License (CC BY 4.0).

http://creativecommons.org/licenses/by/4.0/

(c) (i) Open Access

\begin{abstract}
Cancer is a set of diseases including abnormal growth of cells that can spread to another tissue. Verbascoside (or acteoside) is a naturally occurring, water-soluble secondary metabolite with significant biological properties, which is distributed widely in plant kingdom. Verbascoside is pharmacologically active compounds with many recent evidences that support its biological activities and safety. This review focuses on the recent studies that concerned with the antitumor activities of verbascoside alone and as a synergistic agent as well as nanoproduct. It also shows the latest advances in its antitumor effects, cytotoxic selectivity and its efficiencies in treating cancer, in vitro and/or vivo.
\end{abstract}

\section{Keywords}

Verbascoside, Phenylethanoids, Phenylpropanoids, Glycosides, Cytotoxic, Antitumor

\section{Introduction}

In spite of the significant technological advances in diagnosis and treatment of tumors in the past few decades, cancer (CA) transpires to be one of the key causes of death around the world [1], and considered as a second leading cause of mortality, globally, 9.6 million deaths was recorded by WHO in 2018 [2]. Cancer may harm various organs and occurs in different tissue levels. Prostate, lung colorectal, liver and stomach CAs are the most common kinds of CA in men, while breast, cervical, thyroid, lung, and colorectal CAs are the most common types among women [1]. Moreover, blood CA is the most common CA in children [3]. The common causes of CA are lifestyle parameters (90\% - 95\%) 
like food habits of having carbonated beverages, poor nutrition, smoking, junk food and excess alcohol consumption. In addition to the genetic factors (5\% 10\%) [4]. Now days, when the Corona virus became pandemic, individuals with cancer may be more likely to have Corona virus disease (COVID-19) [5].

Many options are available for cancer treatment starting with surgical removal and radiation treatment of the large accumulated or sized biomass of cancer. The next option to surgery and radiation is treatment with systemic chemotherapy. Chemotherapeutic agents include alkylating agents ((e.g doxorubicin), antimetabolites (e.g., cytrabine), anti-tubulin agents (taxanes), hormones, molecular targeting agents and phytochemicals [5].

Phytochemicals are naturally occurring compounds from plants which serve as vital resources for cancer therapy and production of novel drugs. Many examples of phytochemicals used in cancer treatment include podophyllotoxin analogs, taxol analogs, vinca alkaloids such as vinblastine and vincristine and polyphenols. The most common mechanism of phytochemicals is regulating molecular pathways in cancer cells [6]. Polyphenols have been extensively examined for their possible benefits such as antioxidant, anti-angiogenic, antiproliferative antitumor, hypoglycemic and immunomodulatory properties that have increase in their applications in the functional foods, pharmaceutical and cosmetic industries [7]. Phenolic compounds can rapidly scavenge free radicals like hydroxyl radicals, superoxide radicals, peroxyl radicals, hydrogen peroxide $\left(\mathrm{H}_{2} \mathrm{O}_{2}\right)$, and reactive oxygen species (ROS) by transferring single electron or hydrogen atoms to free radicals. These mechanisms are the main physicochemical factors to evaluate the antioxidant efficiency of plant polyphenols [8].

Although many enhancements have been made, several semi-synthetic or synthetic derivatives of anti-tumor drugs are depend on natural molecules with anti-neoplastic properties. In this field, the most contribution of anti-tumor molecules belongs to plant secondary metabolites, specially phenolic compounds [9]. Plant polyphenols can inhibit CA development by modulation of some of signal transduction pathways that takes place in cancer cells. The key antitumor mechanisms involve modulation of some apoptotic proteins like cyclooxygenase-2, NF- $\mathrm{kB}$, endothelin-1 and STAT3 and modulation in the pro inflammatory cytokines [10]. Various polyphenols are from plant origins representing around 8000 diverse structures. They have at least one or more aromatic rings containing, at least, one or many hydroxyl groups, that connected to glycosides or esters, enhancing their stability. Phenolic compounds include various molecules, like flavonoids, lignans, stilbenes, tannins, and, phenolic acids (hydroxycinnamic acids and hydoxybenzoic acids) [11].

Phenylethanoid-phenylpropanoid glycosides (PPGs) are widely distributed compounds in the kingdom of plants, most of these compounds isolated from medicinal plants, which are soluble in water and organic solvents [12]. This kind of glycosides are not specific to any organ in the plant, they have been isolated from plant leaves, aerial parts, barks, roots, callus and cell cultures [13] [14]. Most 
of these glycosides reported yet, were isolated from Plantaginaceae, Orobanchaceae Scrophulariaceae, Lamiaceae, Oleaceae and Lamiaceae families [15].

The basic structure characterized by 3 parts which are hydroxyphenylethyl (C6-C2) moiety, cinnamic acid moiety (C6-C3) and sugar part (which is usually di or trisaccharide) to which the first two moieties attached by the glycosidic linkage [16] as shown in (Figure 1) [17]. In few past years, attention has been growing about this type of glycosides, verbascoside (VERB) in particular, due to the high number of literatures that define its evident part in the prophylaxis and treatment of different disorders and diseases. [18]

This review was conducted to focus on verbascoside and its in vivo and/or in vitro anticancer activities against different types of tumors or cancer cell lines. It also extends to show the possible anticancer mechanism alone or in combination with other anticancer drugs. In addition, it discusses shortly some new applications of VERB with nanotechnology. Furthermore, this study attracts attention to the high efficiency and safety of VERB in order to enter the clinical field.

\section{Chemistry and Biosynthetic Pathway of Verbascoside (VERB)}

VERB is an important secondary metabolite that distributed in different plant species, consist of four moieties, phenylethyl alcohol, caffeic acid, rhamnose and glucose. The biosynthetic pathway of VERB remains to be completely elucidated. The first steps are well-known, but numerous intermediates and key enzymes with their corresponding genes remain to be unknown. Existing knowledge of this biosynthetic pathway, which is depend on experiments that including the feeding of plant with precursors labelled with specific isotope [19]. The biosynthesis starts with generation of tyrosine and phenylalanine precursors through the shikimic acid pathway [20]. The hydroxytyrosol part of VERB is synthesized from tyrosine through tyramine and also from dopamine, however, its caffeoyl part is biosynthesized from phenylalanine via cinnamic acid pathway [21]. Dopamine molecule is combined into phenylethanoid-phenylpropanoid glycoside by oxidation to aldehyde, then reduction to the alcohol, followed by $\beta$-glycosylation [22]. The sugar part is disaccharide (rhamnose and glucose) the phenylethanoid and phenylpropanoid parts attached to the rhamnose part by ether and

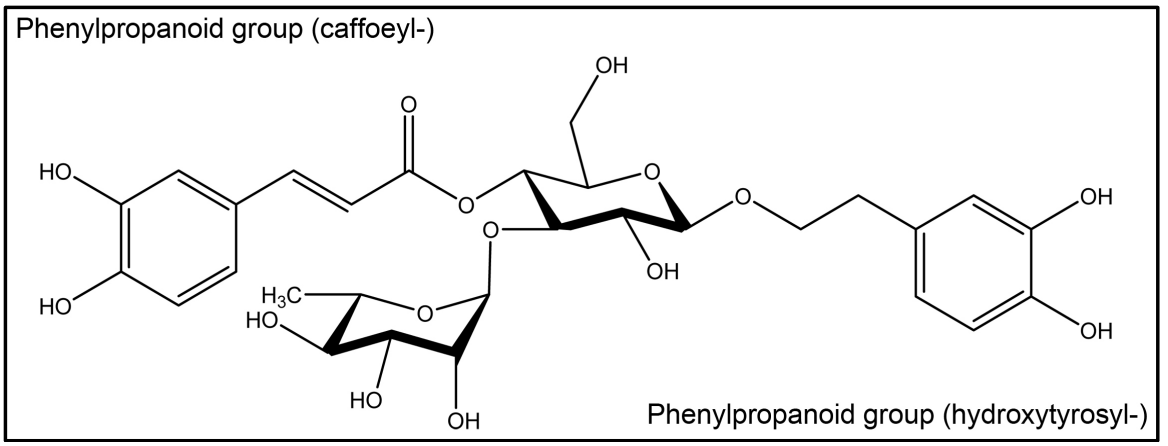

Figure 1. VERB structure. 
ester bond respectively [19].

Many bioactivities of VERB are discovered till now, it has a wide range of pharmacological activities. The plants that are rich in these glycosides known for their powerful antioxidant activities [23]. Many researchers used variety of tumor models to identify the antitumor activities of PPGs. VERB, well-known PPG, which has different in vitro and in vivo clinical activities on various diseases [24] [25], with evidence-supported bioactivities including neuroprotection [26], antidiabetic [27], antioxidant [28], anticancer [29] antiinflammatory, antiandrogen, antimicrobial [30] [31] [32]. Toxicity studies on VERB showed its high level of safety as oral LD50 less than $2000 \mathrm{mg} / \mathrm{kg}$, which give it high level of safety [33].

\section{Anticancer Activities}

VERB showed anticancer activities against many cancer cell lines as the following below.

\subsection{Brain Cancer}

Glioblastoma is one of the most prevalent malignant tumors in gliomas, it constitutes about $45.5 \%$ of primary malignant tumors of brain [34]. SHP-1 is one of tyrosine phosphate protein, which is considered as a unique tumor suppressor gene that involved in different hallmarks of glioblastoma and other types of cancer. The key mechanism by which SHP-1 limits cancer progression and development is the capability to weaken signaling pathways, which control cell proliferation, migration, survival and invasion [35]. SHP-1 activities can be inhibited by small interfering RNA (siRNA) [36].

In 2018, Chinese researchers studied the anticancer activities of VERB by targeting protein tyrosine phosphatase (SHP-1) and STAT3. The result showed lower activity VERB when administered together with si-SHP-1 (siRNA + SHP1) and higher cell migration and invasion in comparison to that shown in the VERB-treated mice [37]. Moreover, the survival rate in the mice group treated with combination of temozolomide (TMZ) and VERB was less than that in the groups treated with VERB or TMZ alone with higher apoptosis. The results indicated that si-SHP-1 could partially inverse the effect of VERB, by decreasing the expression of SHP-1 and increasing the expression of p-STAT3, (p-STAT3 which is often associated with cell survival, proliferation, and transformation) [38]. The results demonstrated that VERB enhanced the TMZ-induced inhibition on U87 cell (brain tumor cell line) invasion and migration [39]. Moreover combination of TMZ with VERB offered a therapeutic capability for treatment of glioblastoma. This synergistic effect of VERB on the therapeutic mechanisms of TMZ was explained through mitogen-activated protein kinases (MAPK) pathway. This synergistic effect also was demonstrated on C6 cell line from rat glioblastoma treated by TMZ, with VERB. Following co-treatment, the results showed higher expression levels of LC3 and LAMP1 (autophagy markers) [40], 
and higher conversion rate of LC3-I to LC3-II (autophagosome generation sign), leading to enhancement of autophagy. Additionally, the combination therapy showed expression levels of p-ERK, P-38 and p-JNK (MAPKs that activated in response to stress stimuli) higher than treatment with TMZ only [37].

\subsection{Colorectal Cancer}

Colorectal cancer (CRC), the second most common cancer in the United States, is associated with a high prevalence of mutations [36]. VERB has high synergistic effect with 5-Fluorouracil (5-Fu) against colorectal cancer cells. Resistance of colorectal cancer cells was previously related to upregulation of AKT/PI3K pathway in these tumor cells [41], and therefore, suppression of this pathway has been suggested for sensitizing cancer cells to conventional treatment. The gene expression of Bcl-xL, p53, Bcl-2, Bax, Akt, PI3K and caspase-3,8,9 were appraised in Caco-2 cells treated with $5-\mathrm{Fu}+$ VERB as a combined treatment. The combined treatment resulted in greater Bax expression level than 5 - Fu $(10 \mu \mathrm{M})$ and VERB $(0.1 \mu \mathrm{M})$ alone. Moreover, VERB + 5-Fu-treated cells showed more than 1 fold lower P-AKT/total AKT ratio than that of the control cells, which suggest an improvement in colon cancer cells to 5-Fu [42]. The combined treatment indicated a significant decrease in PI3K that reached to $89.14 \%$ compared to control cells. In case of the caspases-3, 8 and 9, the combined treatment increased these caspases by 7.1 fold, while the increase was 6.7 fold in case of using 5-Fu only compared to control [43].

In another study, human colorectal cancer LoVo, HCT-116, SW620 and HT29 cell lines were used for in vitro study, and HCT-116 cells were used for in vivo study using nude mice. VERB showed that with increasing doses $(20,40$, and $80 \mathrm{mg} / \mathrm{kg} /$ day), the inhibition rates of tumor weight were $42.79 \%, 53.90 \%$, and $60.99 \%$, respectively. Particularly, at higher dose, the anti-tumor efficiency of VERB was similar to 5-Fu. VERB also improved the expression of Bax, HIPK2 and 553 (pro-apoptotic proteins) in tumors [44], and decreased the expression of Bcl-2 (anti-apoptotic protein) in a dose-dependent manner. When VERB administered together with p53-specific inhibitor (PFT-a) the rate of apoptosis is highly reduced, this strongly proposed that the apoptosis induced by VERB is the result of p53-HIPK2 signaling pathway activation [45].

\subsection{Lung Cancer}

Pulmonary cancer is the primary cause of mortality from the cancer worldwide (18.41\% of cancer deaths) and results in more deaths than colorectal, cervical and breast cancers combined [46] about $15 \%$ of lung cancer patients are remain alive five years after diagnosis, because about $70 \%$ of patients have progressive disease at the diagnosis time [47].

The antitumor mechanisms for VERB on the viability of A549 (adenocarcinomic cell from human alveoli), HT-29 (cancer cell from human colon), and MCF-7 (human breast cancer cell line) cells, and mechanisms included cell cycle 
arrest, modification in apoptosis and intracellular ROS level (that have antitumor effect in high level) were observed [48]. The reduction in the cell viability was concentration-dependent, at $100 \mu \mathrm{g} / \mathrm{mL}$, the cell viability reduced by VERB as $60.9 \%, 65.6 \%, 68.6 \%$ in A549, HT-29 and MCF-7 cells respectively. The IC50 of MCF-7 cell was lower than other cells (i.e. A549 and HT-29), which indicated higher sensitivity of these cells to the antitumor activity of VERB, as shown in (Figure 2). The rate of apoptosis increased by 2.3, 2.5 and 7.5-times in A549, MCF-7 and HT-29 cells respectively in comparison with control cells. VERB also showed an increase in the ROS production in all cancer cells with maximum effects from 1 to 2 hours after treatment with VERB $(100 \mu \mathrm{g} / \mathrm{mL})$, A549 pulmonary cells showed the highest sensitivity, with ROS levels that increased by $9.4 \%, 21.3 \%$, and $37.7 \%$, after 2 , 3, and 24 hrs incubation periods, respectively [49].

\subsection{Liver Cancer}

The largest organ in the human body, the liver, that can be exposed to many conditions and routines (e.g. viral infection, toxins and alcohol abuse) that lead to its inflammation and damage, which can be advanced to liver carcinoma; the excessive production of pro-inflammatory cytokines is the main cause of inflammation and damage of liver [50].

The inhibitory action of VERB against the production of tumor necrosis factor (TNF- $\alpha$ ) and interleukin-6 (IL-6) compared to silymarin was estimated. At first HepG2 cells (cancer cell line from human liver) treated with VERB at 40, 60 and $80 \mu \mathrm{M}$, then the cells treated with alcohol for inducing the cytokines production (i.e. TNF- $\alpha$ and IL-6); the maximum inhibition (in comparison with control) shown at $80 \mu \mathrm{M}$ VERB concentration that is time-independent. The inhibition of IL- 6 and TNF- $\alpha$ by VERB was assessed to be $37.9 \%$ and $28.3 \%$ respectively, while silymarin at $100 \mu \mathrm{M}$ showed $29.9 \%$ and $38.7 \%$ inhibition of

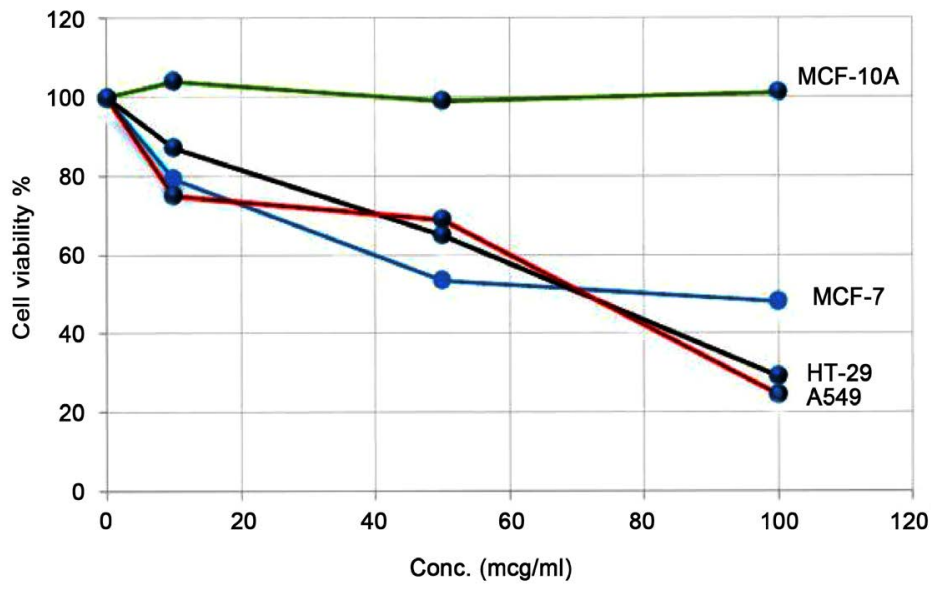

Figure 2. Conc-response curve, which show the $\mathrm{IC}_{50}$ for each type of cells. MCF-7 shows to have higher sensitivity to the treatment with lower $\mathrm{IC}_{50}$, while MCF-10A shows no remarkable changes after treatment, which indicate the high selectivity of VER. 
TNF- $\alpha$ and IL-6 respectively [33].

VERB showed a high efficacy against hepatocellular carcinoma (HCC) after analyzing the proliferation and migration of cells in HLF, JHH-7, and BEL7404 xenografts (liver tumor cell line from human) in mice. Additionally, angiogenesis inhibition by VERB was also analyzed using human umbilical vein endothelial cells (HUVECs). The kallikren-related peptidase (KLK) has been proposed as a tumor biomarker in the diagnosis as well as in the prognosis of different cancers, due to its disturbed expression [51]. VERB also has the ability to exert an antitumor activity in HCC cell lines and in mice transfecting with HUVECs by increasing p53 levels and inhibiting KLK and angiogenesis and also prohibit the cell proliferation in all the three cell lines. However, the prohibition potency together with sorafenib (anticancer) as a co-treatment was stronger than that of sorafenib or VERB alone. Moreover, VERB + sorafenib showed higher wound healing inhibition than single treatment which indicates the high efficiency of VERB in prevention of cell migration. In the case of angiogenesis, which is linked to tumor growth, progression and metastasis, the inhibition of HUVECs migration, alignment and elongation, and formation of vessel-like structures were also highly efficient with co-treatment [52].

\subsection{Breast Cancer}

The VERB effect is shown to be similar to that of the known phytoestrogens such as resveratrol, which indicate that VERB has plant estrogenic action [53]. VERB has anti-breast cancer activity, which could antagonize the upregulation of estrogen response element (ERE) luciferase by estradiol, this because VERB has competitive effect with estradiol by blocking the receptors of estrogen in HeLa cells. VERB interacted with Sterile Alpha Motif Domain 3 (SAMD3), which is protein-coding gene, resulting in its phosphorylation, which in turn could hinder the cell proliferation. Additionally, VERB shown to promote the expression of activator protein 1 (AP-1), which can regulates a number of cell processes, including proliferation, differentiation and apoptosis; activated AP-1 can reduce the gene expression MYC (an oncogene) and CDk6, which is an important key in the progression of the cell cycle [54].

In turkey, some researchers isolated VERB from Phlomis nissolii and studied the ability of VERB for apoptotic induction in the breast cancer cell lines. VERB IC50 for MCF-7 and MDA-MB 231 cell lines after exposure to different concentrations of VERB, as shown in (Table 1).

Table 1. VERB IC50 for MCF-7 and MDA-MB 231 cell lines.

\begin{tabular}{ccc}
\hline $\begin{array}{c}\text { The time of exposure } \\
\text { to VERB }\end{array}$ & IC50 $(\mu \mathrm{M})$ for MCF-7 & $\begin{array}{c}\text { IC50 }(\mu \mathrm{M}) \text { for MDA-MB } \\
231\end{array}$ \\
\hline $24 \mathrm{hr}$ & 0.127 & 0.159 \\
$48 \mathrm{hr}$ & 0.217 & 0.258 \\
$72 \mathrm{hr}$ & 0.282 & 0.256 \\
\hline
\end{tabular}


After using different concentrations of VERB (100, 48, 25, 10, 1, 0.5 and 0.1 $\mu \mathrm{M})$, the result showed that only $100 \mu \mathrm{M}$ was effective with highest cytotoxic activity on MCF-7 after $72 \mathrm{hr}$ and highest cytotoxic activity on MDA-MB 231 after $24 \mathrm{hr}, 48 \mathrm{hr}$ and $72 \mathrm{hr}[55]$.

\subsection{Hematologic Cancer}

The dynamics of hematopoietic stem cell may precede many blood cancers, involving myelodysplastic syndrome, myeloproliferative neoplasms, chronic lymphocytic leukemia and acute myeloid leukemia [56]. Kyung-Won Lee and his group revealed that VERB prevented the growth of HL-60 cells (human leukemia cell line) in a time and concentration-dependent style with an IC50 of 30 mM. Additionally, flow cytometric analysis revealed that VERB blocked progression of cell cycle at the G1 phase in HL-60 cell [57]

Nanotechnology is the use of material on an atomic, molecular or supramolecular scale for different purposes. Medicinally, nanotechnology plays an advance role in enhancing the absorption, bioavailability and efficacy of drugs [58]. It has been shown that nanocarriers loaded with antioxidant can be used in various formulations with a high and controlled-release antioxidant effect that would meet the modern requests of consumers [11]. Recently, Chinese scientists developed a new system for better delivery of VERB for enhancing its chemotherapeutic effect against drug-resistant type of leukemia cells (K562/A02, KA). A novel combination product of poly $\mathrm{n}$-isopropyl acrylamide with gold nanoshells, showed a better role of drug delivery [59]. VERB nanoproduct (200 $\mathrm{mm})$ shown obvious tumor inhibitory effects by increasing the expression of initiator caspases (e.g. caspase 3,8,9) inside the KA cells with smaller size of tumor in comparison with control group and those treated with VERB alone, and the result proved that this delivery system enhance the anticancer activity of VERB [60].

\subsection{Skin Cancer}

Polyphenols of plant origin, known for their antioxidant, anti-inflammatory, antimutagenic, and antiproliferative characteristics in vitro and in vivo [61]. Protective effects of polyphenols in green tea against UVB-induced skin cancer [62], pro-cyanidins in grape seeds [63], curcumin, silymarin and genistein were confirmed to be potent protective agents against initiation and progression steps of UVA and UVB carcinogenesis on the mouse model [64] [65] [66] [67]. However, because of their poor gastrointestinal absorption, little bioavailability, and enhanced metabolism; the clinical chemoprotective worth of VERB and other polyphenols through oral route is still questioned [68].

Moreover, VERB has shown to be more toxic in A5 cells (multistage cancer of mouse skin) than $\mathrm{C} 5 \mathrm{~N}$ cells (non-carcinogenic). It was found that VERB differentially reduced the phosphorylation of ribosomal protein S6 and enhanced the phosphorylation of p53, Creb1, Stat6, Fak1 and pro-apoptotic Stat1 in A5 com- 
pared to C5N cells, as well as, VERB resulted in suppression of the MMP-2 and MMP-9 activities in the A5 cells. This indicate that VERB has evidenced selectivity toward tumor cells [69].

\subsection{Prostate Cancer}

Prostate cancer is one of the most common carcinoma in men [70]. There are many chemotherapeutic agents for treating prostate cancer, which are extremely toxic to the normal tissues [71]. To resolve this problem, co-therapy of a chemotherapeutic agent with a remedy possessed anti-proliferative effect. Several studies have revealed that natural secondary metabolites that have a cinnamic acid moiety possess anti-proliferative effects on tumor cell lines [57].

VERB has ability to promote rat prostate apoptosis, and inhibit benign prostatic hyperplasia (BPH). VERB with high-dose treatment can results in apoptosis in the rat prostate cells, which is significantly higher in comparison with model group [72]. Treatment with VERB significantly prevented cell proliferation, and migration capabilities of men prostate tumor cell line (e.g. PC-3 cells and Du145) by suppression of HMGB1/RAGE pathway, which resulted in downregulation of TGF- $\beta$-associated epithelial-mesenchymal transition (EMT) progression [73].

\section{Miscellaneous}

DNA is under continuous stress, which results from cellular metabolism or environmental factors [74]. Reactive oxygen species (ROS) cause DNA damage through oxidative damage, hence, playing an important role in the initiation of tumor [75]. The oxidative DNA stress has been implicated in the induction of several diseases including inflammation, heart disease and cancer [76].

Abeliophyllum distichum (AAD) is a Korean plant that rich in VERB [77]. A recent Korean study clarified the essential role of AAD in preventing the oxidative damage of DNA. The results showed that AAD eliminated 1,1-Diphenyl-2-picryl hydrazyl (DPPT) and 2,2-Azino-bis (3-ethylbenzothiazoline-6-sulfonic acid) diammonium salt (ABTS) free radicals in dose-dependent manner, IC 50 for AAD and control (L-ascorbic acid) were 8.8 and $5.0 \mu \mathrm{g} / \mathrm{ml}$ respectively in case of DPPT, and 6.47 and $10.49 \mu \mathrm{g} / \mathrm{ml}$, respectively, in case of ABTS. ADD also enhanced the cell viability compared with control cells after exposure to $\mathrm{H}_{2} \mathrm{O}_{2}$-induced damage [78].

VERB considered as strongest antioxidant recognized in Australian olive mill waste [79]. VERB Hydroxytyrosol and oleuropein at $10 \mu \mathrm{M}$ significantly decreased the proliferation of gastric adenocarcinoma (AGS cells) by 19, 27 and $16 \%$, respectively. Though, the ethyl acetate extract of olive mill waste (biophenols extract) was more potent as anti-proliferative than VERB alone [80].

VERB also showed antitumor activity against oral squamous cell carcinoma (OSCC) by decreasing the vitality and metastasis of HN6 and HN4 cancer cells, while stimulating apoptosis. VERB efficiently inhibited activation and downstream 
of nuclear factor (NF)-jB and expression of Bcl-2/Bcl-XL, resulting in high rate of OSCC cell apoptosis, consequently, mRNA and matrix metalloproteinase- 9 expression have been suppressed, thus, VERB inhibiting metastasis of cancer cell [81].

\subsection{VERB and Nanotechnology}

Based on the advantage of nanotechnology in cancer therapeutics in improving the pharmacokinetics and reducing the systemic toxicities of chemotherapies assuming the selective targeting and delivery of these anticancer drugs to tumor tissues [82]. So the beneficial effect of medicinal agent-loaded nanoparticles have become highly concluded [83]. Many researches studies the application of VEBR loaded nanoparticles in treatment of cancer and the results were better than without nanoparticles. VERB carried on nickel nanoparticles showed a synergistic effect on the of apoptosis induction in doxorubicin-resistant human erythroleukemic cell line (K562). Observations demonstrate that nickel have ability to facilitate the VERB uptake into K562 cells. Additionally, in vivo study indicated that the tumor growth in the mice could be efficiently inhibited by these nanoparticles. Thus, VERB-Nickel can serve as a novel approach to sensitively led the tumor cells to effective chemotherapy [77]. VERB carried on gold nanoparticles $\mathrm{Au}$ ) also were studied and demonstrated that VERB-Au nanoparticles provided an effective strategy to control tumor cell growth [29].

\subsection{Safety and Side Effects of VERB}

VERB has a wide range of biological and pharmacological activities so studying its side effects and toxicity is important. [18]. VERB has oral LD50 less than 2000 $\mathrm{mg} / \mathrm{kg}$, which give it high level of safety [33]. A single intraperitoneal VERB injection at 1,2 and $5 \mathrm{~g}$ per $\mathrm{kg}$ did not induce deaths and side effects in mice. Therefore, LD50 value of VERB was found to be greater than $5 \mathrm{~g}$ per $\mathrm{kg}$, and a substance with a LD50 in range of $1-5 \mathrm{~g}$ per $\mathrm{kg}$ is considered low-toxic [84]. Additionally, in vitro, VERB showed no cytotoxic effects on HepG2 and NIH cell at concentrations up to $400 \mu \mathrm{M}$, as well as, VERB did not cause significant changes in hematological and biochemical and histopathological parameters [85]. Further wide studies may be required to set up on the possible adverse effects of VERB.

\section{Conclusions}

This review summarized the latest studies that proved the antitumor activities of VERB. As a polyphenolic compound, VERB showed a powerful antioxidant and antiproliferative efficiencies, alone and as a synergistic agent. VERB has ability to be more effective when given together with chemotherapeutic agent (e.g. TMZ, 5-Fu, cisplatin, sorafenib, etc.). Moreover, lower effective dose of chemotherapeutic agent is needed with co-treatment, and hence, less toxic effects of chemotherapy. For instance, IC50 of 5-Fu was highly reduced from $1.1990 \mu \mathrm{M}$ 
when used as a single treatment to $0.1875 \mu \mathrm{M}$ when combined with $0.1 \mu \mathrm{M}$ VERB. VERB + 5-Fu-treated cells, showed the lowest p-AKT levels, in comparison with VERB, control and 5-Fu-treated cells.

Resistant cells characterized by modified membrane transport, improved DNA repair, defects in apoptotic pathway, modification of proteins, target molecules and pathway mechanisms, like enzymatic deactivation [86]. One way to overcome the multidrug-resistant tumor cell is the nanoproduct, which enhances the bioavailability of anticancer agent inside the tumor cell. VERB nanoproduct significantly stimulated apoptosis-related caspases expression in tumor cells, which might offer a new chemotherapeutic approach in cancer treatment such as leukemia. ROS is like a sword with two edges, at low levels, ROS enhances the survival of tumor cell [87], while at high level, ROS can overwhelm tumor growth by activation of cell cycle inhibitors [88]. Additionally, VERB has high ability to increase ROS inside the tumor cell like A549, HT-29 and MCF-7 that showed time-dependent generation of ROS in tumor cells (1 - 24 hours) [49].

Collective data with the evidences showed that VERB is active compound with high selectivity, no toxicity in animals and no mutagenic effects and it seems to be possible for the future use of the co-therapy of VERB and chemotherapy in clinic [69] [89]. Also loading of VERB on nanoparticles can provide a good tool for delivery of VERB to cancer sites [9].

In spite of the fortune of laboratory data, which is accessible that describe the anti-tumor activities of VERB after in vitro results additional to the animal models, many questions remain unsettled with respect to actual clinical applications. Evidence-based human researches in large scale with precise therapeutic settings are important. More intensive studies are necessary to settle the clinical potential of VERB, thus permitting its acceptance as a therapeutic compound. VERB is also has a special structure that suggested an interesting scaffold with many reactive sites for combinatorial chemistry.

\section{Conflicts of Interest}

The authors declare no conflicts of interest regarding the publication of this paper.

\section{References}

[1] Miller, K.D., Siegel, R.L., Lin, C.C., Mariotto, A.B., Kramer, J.L., Rowland, J.H., Stein, K.D., Alteri, R. and Jemal, A. (2016) Cancer Treatment and Survivorship Statistics, 2016. CA: A Cancer Journal for Clinicians, 66, 271-289. https://doi.org/10.3322/caac.21349

[2] World Health Organization (WHO) (2020) Cancer. https://www.who.int/health-topics/cancer\#tab=tab 1

[3] Lee, S.H. and Ham, E.M. (2010) The Relationship between the Optimistic Bias about Cancer and Cancer Preventive Behavior of the Korean, Chinese, American, and Japanese Adult Residing in Korea. Journal of Korean Academy of Nursing, 40, 52 59. https://doi.org/10.4040/jkan.2010.40.1.52 
[4] Anand, P., Kunnumakara, A.B., Sundaram, C., Harikumar, K.B., Tharakan, S.T., Lai, O.S., Sung, B. and Aggarwal, B.B. (2008) Cancer Is a Preventable Disease That Requires Major Lifestyle Changes. Pharmaceutical Research, 25, 2097-2116. https://doi.org/10.1007/s11095-008-9661-9

[5] Tian, Y., Qiu, X., Wang, C., Zhao, J., Jiang, X., Niu, W., Huang, J. and Zhang, F. (2021) Cancer Associates with Risk and Severe Events of COVID-19: A Systematic Review and Meta-Analysis. International Journal of Cancer, 148, 363-374.

https://doi.org/10.1002/ijc.33213

[6] Nussbaumer, S., Bonnabry, P., Veuthey, J.L. and Fleury-Souverain, S. (2011) Analysis of Anticancer Drugs: A Review. Talanta, 85, 2265-2289. https://doi.org/10.1016/j.talanta.2011.08.034

[7] Rostamabadi, H., Falsafi, S.R. and Jafari, S.M. (2019) Nanoencapsulation of Carotenoids within Lipid-Based Nanocarriers. Journal of Controlled Release, 298, 38-67. https://doi.org/10.1016/j.jconrel.2019.02.005

[8] Leopoldini, M., Russo, N. and Toscano, M. (2011) The Molecular Basis of Working Mechanism of Natural Polyphenolic Antioxidants. Food Chemistry, 125, 288-306. https://doi.org/10.1016/j.foodchem.2010.08.012

[9] Jafari, S.M. and McClements, D.J. (2017) Nanotechnology Approaches for Increasing Nutrient Bioavailability. Advances in Food and Nutrition Research, 81, 1-30. https://doi.org/10.1016/bs.afnr.2016.12.008

[10] Zare, M., Norouzi Roshan, Z., Assadpour, E. and Jafari, S.M. (2021) Improving the Cancer Prevention/Treatment Role of Carotenoids through Various Nano-Delivery Systems. Critical Reviews in Food Science and Nutrition, 61, 522-534. https://doi.org/10.1080/10408398.2020.1738999

[11] Maqsoudlou, A., Assadpour, E., Mohebodini, H. and Jafari, S.M. (2020) Improving the Efficiency of Natural Antioxidant Compounds via Different Nanocarriers. Advances in Colloid and Interface Science, 278, Article ID: 102122. https://doi.org/10.1016/j.cis.2020.102122

[12] de Souza Gil, E., Adrian Enache, T. and Maria Oliveira-Brett, A. (2013) Redox Behaviour of Verbascoside and Rosmarinic Acid. Combinatorial Chemistry \& High Throughput Screening, 16, 92-97. https://doi.org/10.2174/138620713804806337

[13] Oyourou, J.N., Combrinck, S., Regnier, T. and Marston, A. (2013) Purification, Stability and Antifungal Activity of Verbascoside from Lippia javanica and Lantana camara Leaf Extracts. Industrial Crops and Products, 43, 820-826. https://doi.org/10.1016/j.indcrop.2012.08.028

[14] Fan, Y., Xu, C., Li, J., Zhang, L., Yang, L., Zhou, Z., Zhu, Y. and Zhao, D. (2018) Ionic Liquid-Based Microwave-Assisted Extraction of Verbascoside from Rehmannia Root. Industrial Crops and Products, 124, 59-65.

https://doi.org/10.1016/j.indcrop.2018.07.063

[15] Taskova, R.M., Gotfredsen, C.H. and Jensen, S.R. (2005) Chemotaxonomic Markers in Digitalideae (Plantaginaceae). Phytochemistry, 66, 1440-1447.

https://doi.org/10.1016/j.phytochem.2005.04.020

[16] Dembitsky, V.M. (2005) Astonishing Diversity of Natural Surfactants: 5. Biologically Active Glycosides of Aromatic Metabolites. Lipids, 40, 869-900. https://doi.org/10.1007/s11745-005-1449-2

[17] Hasan, A.K., Ibrahim, S.A., Amani, A.T. and Monther, F.M. (2018) Determination, Isolation, and Identification of Aucubin and verbascoside in the Leaves of Iraqi Plantago lancoleta L. Using Different Detecting Methods. International Journal of Pharmacy and Pharmaceutical Sciences, 11, 74-80. 
[18] Alipieva, K., Korkina, L., Orhan, I.E. and Georgiev, M.I. (2014) Verbascoside-A Review of Its Occurrence, (Bio)Synthesis and Pharmacological Significance. Biotechnology Advances, 32, 1065-1076. https://doi.org/10.1016/j.biotechadv.2014.07.001

[19] Zhou, Y., Zhu, J., Shao, L. and Guo, M. (2020) Current Advances in Acteoside Biosynthesis Pathway Elucidation and Biosynthesis. Fitoterapia, 142, Article ID: 104495. https://doi.org/10.1016/j.fitote.2020.104495

[20] Świeca, M. (2016) Hydrogen Peroxide Treatment and the Phenylpropanoid Pathway Precursors Feeding Improve Phenolics and Antioxidant Capacity of Quinoa Sprouts via an Induction of L-Tyrosine and L-Phenylalanine Ammonia-Lyases Activities. Journal of Chemistry, 2016, Article ID: 1936516.

https://doi.org/10.1155/2016/1936516

[21] Ellis, B.E. (1983) Production of Hydroxyphenylethanol Glycosides in Suspension Cultures of Syringa vulgaris. Phytochemistry, 22, 1941-1943. https://doi.org/10.1016/0031-9422(83)80018-1

[22] Saimaru, H. and Orihara, Y. (2010) Biosynthesis of Acteoside in Cultured Cells of Olea europaea. Journal of Natural Medicines, 64, 139-145.

https://doi.org/10.1007/s11418-009-0383-Z

[23] Eldesoky, A.H., Abdel-Rahman, R.F., Ahmed, O.K., Soliman, G.A., Saeedan, A.S., Elzorba, H.Y., Elansary, A.A. and Hattori, M. (2018) Antioxidant and Hepatoprotective Potential of Plantago major Growing in Egypt and Its Major Phenylethanoid Glycoside, Acteoside. Journal of Food Biochemistry, 42, e12567. https://doi.org/10.1111/jfbc.12567

[24] Li, M., Zhou, F., Xu, T., Song, H. and Lu, B. (2018) Acteoside Protects against 6OHDA-Induced Dopaminergic Neuron Damage via Nrf2-ARE Signaling Pathway. Food and Chemical Toxicology, 119, 6-13. https://doi.org/10.1016/j.fct.2018.06.018

[25] Yang, L., Zhang, B., Liu, J., Dong, Y., Li, Y., Li, N., Zhao, X., Snooks, H., Hu, C. and Ma, X. (2019) Protective Effect of Acteoside on Ovariectomy-Induced Bone Loss in Mice. International Journal of Molecular Sciences, 20, Article No. 2974. https://doi.org/10.3390/ijms20122974

[26] Wu, C.J., Chien, M.Y., Lin, N.H., Lin, Y.C., Chen, W.Y., Chen, C.H. and Tzen, J.T. (2019) Echinacoside Isolated from Cistanche tubulosa Putatively Stimulates Growth Hormone Secretion via Activation of the Ghrelin Receptor. Molecules, 24, Article No. 720. https://doi.org/10.3390/molecules24040720

[27] Hernández-Chávez, G., Martinez, A. and Gosset, G. (2019) Metabolic Engineering Strategies for Caffeic Acid Production in Escherichia coli. Electronic Journal of Biotechnology, 38, 19-26. https://doi.org/10.1016/j.ejbt.2018.12.004

[28] Vertuani, S., Beghelli, E., Scalambra, E., Malisardi, G., Copetti, S., Toso, R.D., Baldisserotto, A. and Manfredini, S. (2011) Activity and Stability Studies of Verbascoside, a Novel Antioxidant, in Dermo-Cosmetic and Pharmaceutical Topical Formulations. Molecules, 16, 7068-7080. https://doi.org/10.3390/molecules16087068

[29] Chen, M., Zhang, Y., Huang, B., Yang, X., Wu, Y., Liu, B., Yuan, Y. and Zhang, G. (2013) Evaluation of the Antitumor Activity by Ni Nanoparticles with Verbascoside. Journal of Nanomaterials, 2013, Article ID 623497.

https://doi.org/10.1155/2013/623497

[30] Jing, W., Chunhua, M. and Shumin, W. (2015) Effects of Acteoside on Lipopolysaccharide-Induced Inflammation in Acute Lung Injury via Regulation of NF- $\kappa \mathrm{B}$ Pathway in Vivo and in Vitro. Toxicology and Applied Pharmacology, 285, 128-135. 
https://doi.org/10.1016/j.taap.2015.04.004

[31] Georgiev, M.I., Eibl, R. and Zhong, J.J. (2013) Hosting the Plant Cells in Vitro: Recent Trends in Bioreactors. Applied Microbiology and Biotechnology, 97, 3787-800. https://doi.org/10.1007/s00253-013-4817-x

[32] Azimi, H., Fallah-Tafti, M., Khakshur, A.A. and Abdollahi, M. (2012) A Review of Phytotherapy of Acne Vulgaris: Perspective of New Pharmacological Treatments. Fitoterapia, 83, 1306-1317. https://doi.org/10.1016/j.fitote.2012.03.026

[33] Khullar, M., Sharma, A., Wani, A., Sharma, N., Chandan, B.K., Kumar, A. and Ahmed, Z. (2019) Acteoside Ameliorates Inflammatory Responses through NFkB Pathway in Alcohol Induced Hepatic Damage. International Immunopharmacology, 69, 109117. https://doi.org/10.1016/j.intimp.2019.01.020

[34] Ostrom, Q.T., Gittleman, H., Liao, P., Rouse, C., Chen, Y., Dowling, J., Wolinsky, Y., Kruchko, C. and Barnholtz-Sloan, J. (2014) CBTRUS Statistical Report: Primary Brain and Central Nervous System Tumors Diagnosed in the United States in 20072011. Neuro-Oncology, 16, iv1-iv63. https://doi.org/10.1093/neuonc/nou223

[35] Varone, A., Spano, D. and Corda, D. (2020) Shp1 in Solid Cancers and Their Therapy. Frontiers in Oncology, 10, Article No. 935.

https://doi.org/10.3389/fonc.2020.00935

[36] Yin, S., Wu, H., Lv, J., Wu, X., Zhang, Y., Du, J. and Zhang, Y. (2014) SHP-1 Arrests Mouse Early Embryo Development through Downregulation of Nanog by Dephosphorylation of STAT3. PLOS ONE, 9, e86330.

https://doi.org/10.1371/journal.pone.0086330

[37] Hwang, T.W., Kim, D.H., Kim, D.B., Jang, T.W., Kim, G.H., Moon, M., Yoon, K.A., Choi, D.E., Park, J.H. and Kim, J.J. (2019) Synergistic Anticancer Effect of Acteoside and Temozolomide-Based Glioblastoma Chemotherapy. International Journal of Molecular Medicine, 43, 1478-1486. https://doi.org/10.3892/ijmm.2019.4061

[38] He, S., Liao, G., Liu, Y., Huang, L., Kang, M. and Chen, L. (2015) Overexpression of STAT3/pSTAT3 Was Associated with Poor Prognosis in Gastric Cancer: A Meta-Analysis. International Journal of Clinical and Experimental Medicine, 8, 2001420023.

[39] Jia, W.Q., Wang, Z.T., Zou, M.M., Lin, J.H., Li, Y.H., Zhang, L. and Xu, R.X. (2018) Verbascoside Inhibits Glioblastoma Cell Proliferation, Migration and Invasion While Promoting Apoptosis through Upregulation of Protein Tyrosine Phosphatase SHP1 and Inhibition of STAT3 Phosphorylation. Cellular Physiology and Biochemistry, 47, 1871-1882. https://doi.org/10.1159/000491067

[40] Pugsley, H.R. (2017) Quantifying Autophagy: Measuring LC3 Puncta and Autolysosome Formation in Cells Using Multispectral Imaging Flow Cytometry. Methods, 112, 147-156. https://doi.org/10.1016/j.ymeth.2016.05.022

[41] Bahrami, A., Khazaei, M. and Hasanzadeh, M. (2018) Therapeutic Potential of Targeting PI3K/AKT Pathway in Treatment of Colorectal Cancer: Rational and Progress. Journal of Cellular Biochemistry, 119, 2460-2469.

https://doi.org/10.1002/jcb.25950

[42] McCubrey, J.A., Steelman, L.S., Chappell, W.H., Sun, L., Davis, N.M., Abrams, S.L., et al. (2012) Advances in Targeting Signal Transduction Pathways. Oncotarget, 3 , 1505-1521. https://doi.org/10.18632/oncotarget.802

[43] Attia, Y.M., El-Kersh, D.M., Wagdy, H.A. and Elmazar, M.M. (2018) Verbascoside: Identification, Quantification, and Potential Sensitization of Colorectal Cancer Cells to 5-FU by Targeting PI3K/AKT Pathway. Scientific Reports, 8, Article No. 16939. https://doi.org/10.1038/s41598-018-35083-2 
[44] Puca, R., Nardinocchi, L., Sacchi, A., Rechavi, G., Givol, D. and D’Orazi, G. (2009) HIPK2 Modulates p53 Activity towards Pro-apoptotic Transcription. Molecular Cancer, 8, Article No. 85. https://doi.org/10.1186/1476-4598-8-85

[45] Zhou, L., Feng, Y., Jin, Y., Liu, X., Sui, H., Chai, N., Chen, X., Liu, N., Wang, Y. and Li, Q. (2014) Verbascoside Promotes Apoptosis by Regulating HIPK2-p53 Signaling in Human Colorectal Cancer. BMC Cancer, 14, Article No. 747. https://doi.org/10.1186/1471-2407-14-747

[46] Bray, F., Ferlay, J., Soerjomataram, I., Siegel, R.L., Torre, L.A. and Jemal, A. (2018) Global Cancer Statistics 2018: GLOBOCAN Estimates of Incidence and Mortality Worldwide for 36 Cancers in 185 Countries. CA: A Cancer Journal for Clinicians, 68, 394-424. https://doi.org/10.3322/caac.21492

[47] Siegel, R.L., Miller, K.D. and Jemal, A. (2016) Cancer Statistics, 2016. CA: A Cancer Journal for Clinicians, 66, 7-30. https://doi.org/10.3322/caac.21332

[48] Reczek, C.R. and Chandel, N.S. (2018) ROS Promotes Cancer Cell Survival through Calcium Signaling. Cancer Cell, 33, 949-951.

https://doi.org/10.1016/j.ccell.2018.05.010

[49] Vasincu, A., Neophytou, C.M., Luca, S.V., Skalicka-Woźniak, K., Miron, A. and Constantinou, A.I. (2020) 6-O-(3", 4"-di-O-trans-cinnamoyl)-a-l-rhamno-pyranosylcatalpol and Verbascoside: Cytotoxicity, Cell Cycle Kinetics, Apoptosis, and ROS Production Evaluation in Tumor cells. Journal of Biochemical and Molecular Toxicology, 7, e22443. https://doi.org/10.1002/jbt.22443

[50] Abu-Serie, M.M. and Habashy, N.H. (2019) Two Purified Proteins from Royal Jelly with in Vitro Dual Anti-Hepatic Damage Potency: Major Royal Jelly Protein 2 and Its Novel Isoform X1. International Journal of Biological Macromolecules, 128, 782795. https://doi.org/10.1016/j.ijbiomac.2019.01.210

[51] Kontos, C.K., Mavridis, K., Talieri, M., Scorilas, A. (2013) Kallikrein-Related Peptidases (KLKs) in Gastrointestinal Cancer: Mechanistic and Clinical Aspects. Thrombosis and Haemostasis, 110, 450-457. https://doi.org/10.1160/TH12-11-0791

[52] Ma, D., Wang, J., Liu, L., Chen, M. and Wang, Z. (2020) Acteoside as a Potential Therapeutic Option for Primary Hepatocellular Carcinoma: a Preclinical Study. BMC Cancer, 20, Article No. 936. https://doi.org/10.1186/s12885-020-07447-3

[53] Bowers, J.L., Tyulmenkov, V.V., Jernigan, S.C. and Klinge, C.M. (2000) Resveratrol Acts as a Mixed Agonist/Antagonist for Estrogen Receptors $\alpha$ and $\beta$. Endocrinology, 141, 3657-3667. https://doi.org/10.1210/endo.141.10.7721

[54] Zhang, B., Zheng, R. and Wang, Y. (2020) Study on the Antibreast Cancer Mechanism and Bioactive Components of $\mathrm{Si}$ - Wu-Tang by Cell Type-Specific Molecular Network. Evidence-Based Complementary and Alternative Medicine, 2020, Article ID: 2345970. https://doi.org/10.1155/2020/2345970

[55] Şenol, H. (2019) Cytotoxic Effect and Apoptosis Induction of Verbascoside in MCF7 and MDA-MB-231. Doctoral Dissertation, Near East University, Nicosia.

[56] Genovese, G., Kähler, A.K., Handsaker, R.E., Lindberg, J., Rose, S.A., Bakhoum, S.F., Chambert, K., Mick, E., Neale, B.M., Fromer, M., Purcell, S.M., et al. (2014) Clonal Hematopoiesis and Blood-Cancer Risk Inferred from Blood DNA Sequence. New England Journal of Medicine, 371, 2477-2487. https://doi.org/10.1056/NEJMoa1409405

[57] Lin, H.P., Jiang, S.S. and Chuu, C.P. (2012) Caffeic Acid Phenethyl Ester Causes p $21^{\text {Cip1 }}$ Induction, Akt Signaling Reduction, and Growth Inhibition in PC-3 Human Prostate Cancer Cells. PLoS ONE, 7, e31286.

https://doi.org/10.1371/journal.pone.0031286 
[58] Surendiran, A., Sandhiya, S., Pradhan, S.C. and Adithan, C. (2009) Novel Applications of Nanotechnology in Medicine. Indian Journal of Medical Research, 130, 689-701.

[59] Jiang, C., Li, H., Jia, X., Ma, X., Qian, Y. and Qian, W. (2010) Fabrication and Characterization of Poly (N-isopropyl acrylamide)-Gold Nanoshell Structures. Journal of Nanoscience and Nanotechnology, 10, 6599-605.

https://doi.org/10.1166/jnn.2010.2540

[60] Ma, Z., Zhao, X., Jiang, C., Yu, J., Wu, J. and Zeng, X. (2016) Gold Nanoshells with Verbascoside Induce the Apoptosis of Drug-Resistant Leukemia Cells through Caspases Pathway and Inhibit Tumor Growth. Journal of Nanoscience and Nanotechnology, 16, 7118-7124. https://doi.org/10.1166/jnn.2016.11357

[61] Afaq, F. and Katiyar, S.K. (2011) Polyphenols: Skin Photoprotection and Inhibition of Photocarcinogenesis. Mini Reviews in Medicinal Chemistry, 11, 1200-1215.

[62] Wang, Z.Y., Agarwal, R., Bickers, D.R. and Mukhtar H. (1991) Protection against Ultraviolet B Radiation-Induced Photocarcinogenesis in Hairless Mice by Green Tea Polyphenols. Carcinogenesis, 12, 1527-1530. https://doi.org/10.1093/carcin/12.8.1527

[63] Mittal, A., Elmets, C.A. and Katiyar, S.K. (2003) Dietary Feeding of Proanthocyanidins from Grape Seeds Prevents Photocarcinogenesis in SKH-1 Hairless Mice: Relationship to Decreased Fat and Lipid Peroxidation. Carcinogenesis, 24, 1379-1388. https://doi.org/10.1093/carcin/bgg095

[64] Wright, T.I., Spencer, J.M. and Flowers, F.P. (2006) Chemoprevention of Nonmelanoma Skin Cancer. Journal of the American Academy of Dermatology, 54, 933 946. https://doi.org/10.1016/j.jaad.2005.08.062

[65] Aziz, M.H., Reagan-Shaw, S., Wu, J., Longley, B.J. and Ahmad, N. (2005) Chemoprevention of Skin Cancer by Grape Constituent Resveratrol: Relevance to Human Disease? The FASEB Journal, 19, 1193-1195. https://doi.org/10.1096/fj.04-3582fje

[66] Katiyar, S.K. (2007) UV-Induced Immune Suppression and Photocarcinogenesis: Chemoprevention by Dietary Botanical Agents. Cancer letters, 255, 1-11. https://doi.org/10.1016/j.canlet.2007.02.010

[67] Korkina, L.G., De Luca, C., Kostyuk, V.A. and Pastore, S. (2009) Plant Polyphenols and Tumors: From Mechanisms to Therapies, Prevention, and Protection against Toxicity of Anti-Cancer Treatments. Current Medicinal Chemistry, 16, 3943-3965.

[68] Nichols, J.A. and Katiyar, S.K. (2010) Skin Photoprotection by Natural Polyphenols: Anti-Inflammatory, Antioxidant and DNA Repair Mechanisms. Archives of Dermatological Research, 302, 71-83. https://doi.org/10.1007/s00403-009-1001-3

[69] Cheimonidi, C., Samara, P., Polychronopoulos, P., Tsakiri, E.N., Nikou, T., Myrianthopoulos, V., Sakellaropoulos, T., Zoumpourlis, V., Mikros, E., Papassideri, I., Argyropoulou, A. (2018) Selective Cytotoxicity of the Herbal Substance Acteoside against Tumor Cells and Its Mechanistic Insights. Redox Biology, 16, 169-178. https://doi.org/10.1016/j.redox.2018.02.015

[70] Abate-Shen, C. and Shen, M.M. (2002) Mouse Models of Prostate Carcinogenesis. Trends in Genetics, 18, S1-S5. https://doi.org/10.1016/S0168-9525(02)02683-5

[71] Van Bokhoven, A., Varella-Garcia, M., Korch, C., Johannes, W.U, Smith, E.E., Miller, H.L., Nordeen, S.K., Miller, G.J. and Lucia, M.S. (2003) Molecular Characterization of Human Prostate Carcinoma Cell Lines. The Prostate, 57, 205-225. https://doi.org/10.1002/pros.10290

[72] Sun, W.D., Chen, F. and Sun, Y. (2008) The Pharmacological Research of Acteoside 
on the Action Inhibited Begnin Prostatic Hyperplasia in Rats. Journal of Yangzhou University (Agricultural and Life Science Edition), Issue 4, 33-36.

[73] Wu, C.H., Chen, C.H., Hsieh, P.F., Lee, Y.H., Kuo, W.W. and Wu, R.C. (2021) Verbascoside Inhibits the Epithelial-Mesenchymal Transition of Prostate Cancer Cells through High-Mobility Group Box 1/Receptor for Advanced Glycation EndProducts/TGF- $\beta$ Pathway. Environmental Toxicology, 36, 1080-1089. https://doi.org/10.1002/tox.23107

[74] Schärer, O.D. (2003) Chemistry and Biology of DNA Repair. Angewandte Chemie International Edition, 42, 2946-2974. https://doi.org/10.1002/anie.200200523

[75] Han, H.M., Kwon, Y.S. and Kim, M.J. (2016) Antioxidant and Antiproliferative Activity of Extracts from Water Chestnut (Trapa japonica Flerow). Korean Journal of Medicinal Crop Science, 24, 14-20. https://doi.org/10.7783/KJMCS.2016.24.1.14

[76] Gilgun-Sherki, Y., Rosenbaum, Z., Melamed, E. and Offen, D. (2002) Antioxidant Therapy in Acute Central Nervous System Injury: Current State. Pharmacological Reviews, 54, 271-284. https://doi.org/10.1124/pr.54.2.271

[77] Park, J., Xi, H., Han, J., Lee, J. and Kim, Y. (2020) Prediction and Identification of Biochemical Pathway of Acteoside from Whole Genome Sequences of Abeliophyllum Distichum Nakai, Cultivar Ok Hwang 1ho. Journal of Convergence for Information Technology, 10, 76-91. https://doi.org/10.22156/CS4SMB.2020.10.03.076

[78] Jang, T.W., Choi, J.S. and Park, J.H. (2020) Protective and Inhibitory Effects of Acteoside from Abeliophyllum Distichum Nakai against oxidative DNA Damage. Molecular Medicine Reports, 22, 2076-2084. https://doi.org/10.3892/mmr.2020.11258

[79] Obied, H.K., Prenzler, P.D. and Robards, K. (2008) Potent Antioxidant Biophenols from Olive Mill Waste. Food Chemistry, 111, 171-178. https://doi.org/10.1016/j.foodchem.2008.03.058

[80] Konczak, I., Obied, H.K., Prenzler, P.D., Rehman, A.U. and Robards, K. (2009) Chemistry and Bioactivity of Olive Biophenols in Some Antioxidant and Antiproliferative in Vitro Bioassays. Chemical Research in Toxicology, 22, 227-234. https://doi.org/10.1021/tx8004168

[81] Zhang, Y., Yuan, Y., Wu, H., Xie, Z., Wu, Y., Song, X., Wang, J., Shu, W., Xu, J., Liu, B. and Wan, L. (2018) Effect of Verbascoside on Apoptosis and Metastasis in Human Oral Squamous Cell Carcinoma. International Journal of Cancer, 143, 980 991. https://doi.org/10.1002/ijc.31378

[82] Sindhwani, S. and Chan, W.C. (2021) Nanotechnology for Modern Medicine: Next Step towards Clinical Translation. Journal of Internal Medicine, 289, 1-136. https://doi.org/10.1111/joim.13254

[83] Sim, S. and Wong, N.K. (2021) Nanotechnology and Its Use in imaging and Drug Delivery (Review). Biomedical Reports, 14, Article No. 42. https://doi.org/10.3892/br.2021.1418

[84] Hayes, A.W. and Loomis, T.A. (1996) Loomis's Essentials of Toxicology. Elsevier, Amsterdam.

[85] Etemad, L., Zafari, R., Vahdati-Mashhadian, N., Adel Moallem, S., Shirvan, Z.O. and Hosseinzadeh, H. (2015) Acute, Sub-Acute and Cell Toxicity of Verbascoside. Research Journal of Medicinal Plant, 9, 354-360. https://doi.org/10.3923/rimp.2015.354.360

[86] Alfarouk, K.O., Stock, C.M., Taylor. S., Walsh, M., Muddathir, A.K., Verduzco, D., Bashir, A.H., Mohammed, O.Y., Elhassan, G.O., Harguindey, S. and Reshkin, S.J. (2015) Resistance to Cancer Chemotherapy: Failure in Drug Response from ADME to P-gp. Cancer Cell International, 15, Article No. 71. 
https://doi.org/10.1186/s12935-015-0221-1

[87] Irani, K., Xia, Y., Zweier, J.L., Sollott, S.J., Der, C.J. and Fearon, E.R. (1997) Mitogenic Signaling Mediated by Oxidants in Ras-Transformed Fibroblasts. Science, 275, 1649-1652. https://doi.org/10.1126/science.275.5306.1649

[88] Takahashi, A., Ohtani, N., Yamakoshi, K., Iida, S.I. and Tahara, H. (2006) Mitogenic Signalling and the p16 INK4a-Rb Pathway Cooperate to Enforce Irreversible Cellular Senescence. Nature Cell Biology, 8, 1291-1297.

https://doi.org/10.1038/ncb1491

[89] Lu, B., Li, M., Zhou, F., Huang, W., Jiang, Y., Mao, S., Zhao, Y. and Lou, T. (2016) The Osmanthus fragrans Flower Phenylethanoid Glycoside-Rich Extract: Acute and Subchronic Toxicity Studies. Journal of Ethnopharmacology, 187, 205-212. https://doi.org/10.1016/j.jep.2016.04.049 